\title{
Classical and Relativistic Flux of Energy Conservation in Astrophysical Jets
}

\author{
Lorenzo Zaninetti \\ Physics Department, Turin, Italy \\ Email:zaninetti@ph.unito.it
}

Received 5 November 2015; accepted 4 January 2016; published 7 January 2016

Copyright (C) 2016 by author and Scientific Research Publishing Inc.

This work is licensed under the Creative Commons Attribution International License (CC BY).

http://creativecommons.org/licenses/by/4.0/

(c) (i) Open Access

\begin{abstract}
The conservation of the energy flux in turbulent jets which propagate in the intergalactic medium (IGM) allows deducing the law of motion in the classical and relativistic cases. Three types of IGM are considered: constant density, hyperbolic and inverse power law decrease of density. An analytical law for the evolution of the magnetic field along the radio-jets is deduced using a linear relation between the magnetic pressure and the rest density. Astrophysical applications are made to the centerline intensity of synchrotron emission in NGC315 and to the magnetic field of 3C273.
\end{abstract}

\section{Keywords}

\section{Galaxies: Jets Relativity}

\section{Introduction}

The analysis of turbulent jets in the laboratory offers the possibility of applying the theory of turbulence to some well defined experiments, see [1] [2]. The experiments of Reynolds can be seen in [4]. Analytical results for the theory of turbulent jets can be found in [4]-[7]. Recently the analogy between laboratory jets and extragalactic radio-jets has been pointed out, see [8] [9]. We briefly recall that the theory of "round turbulent jets" can be defined in terms of the velocity at the nozzle, the diameter of the nozzle, and the viscosity, see Section 5 in [6]; as an example the gradients in pressure are not considered. The application of the theory of turbulence to extragalactic radio-jets produces a great number of questions to be solved because we do not observe the turbulent phenomena but the radio features which have properties similar to the laboratory's turbulent jets, i.e. similar opening angles. We now pose the following questions.

- Is it possible to apply the conservation of the flux of energy in order to derive the equation of motion for radio-jets in the cases of constant and variable density of the surrounding medium?

- Can we extend the conservation of the flux of energy to the relativistic regime? 
- Can we model the behaviour of the magnetic field and the intensity of synchrotron emission as functions of the distance from the parent nucleus?

- Can we model the back reaction on the equation of motion for turbulent jets due to radiative losses?

In order to answer these questions, we derive the differential equations which model the classical and relativistic conservation of the energy flux for a turbulent jet in the presence of different types of medium, see Sections 2 and 3. Section 4 presents classical and relativistic parametrizations of the radiative losses as well as the evolution of the magnetic field.

\section{Energy Conservation}

The conservation of the energy flux in a turbulent jet requires the perpendicular section to the motion along the Cartesian $x$-axis, $A$

$$
A(r)=\pi r^{2}
$$

where $r$ is the radius of the jet. The section $A$ at position $x_{0}$ is

$$
A\left(x_{0}\right)=\pi\left(x_{0} \tan \left(\frac{\alpha}{2}\right)\right)^{2}
$$

where $\alpha$ is the opening angle and $x_{0}$ is the initial position on the $x$-axis. At position $x$ we have

$$
A(x)=\pi\left(x \tan \left(\frac{\alpha}{2}\right)\right)^{2} .
$$

The conservation of energy flux states that

$$
\frac{1}{2} \rho\left(x_{0}\right) v_{0}^{3} A\left(x_{0}\right)=\frac{1}{2} \rho(x) v(x)^{3} A(x)
$$

where $v(x)$ is the velocity at position $x$ and $v_{0}\left(x_{0}\right)$ is the velocity at position $x_{0}$, see Formula A28 in [10].

The selected physical units are pc for length and yr for time; with these units, the initial velocity $v_{0}$ is expressed in $\mathrm{pc} \cdot \mathrm{yr}^{-1}, 1 \mathrm{yr}=365.25$ days. When the initial velocity is expressed in $\mathrm{km} \cdot \mathrm{s}^{-1}$, the multiplicative factor $1.02 \times 10^{-6}$ should be applied in order to have the velocity expressed in $\mathrm{pc}^{\cdot} \mathrm{yr}^{-1}$.

\subsection{Constant Density}

In the case of constant density of the intergalactic medium (IGM) along the $x$-direction, the law of conservation of the energy flux, as given by Equation (4), can be written as a differential equation

$$
\left(\frac{\mathrm{d}}{\mathrm{d} t} x(t)\right)^{3}(x(t))^{2}-v_{0}^{3} x_{0}^{2}=0 .
$$

The analytical solution of the previous differential equation can be found by imposing $x=x_{0}$ at $t=0$,

$$
x(t)=\frac{1}{3} 3^{2 / 5} \sqrt[5]{x_{0}^{2}\left(5 t v_{0}+3 x_{0}\right)^{3}} .
$$

The asymptotic approximation is

$$
x(t) \sim \frac{1}{3} 3^{2 / 5} 5^{3 / 5} \sqrt[5]{v_{0}^{3} x_{0}^{2}} t^{3 / 5} .
$$

The velocity is

$$
v(t)=\frac{3^{2 / 5} x_{0}^{2}\left(5 t v_{0}+3 x_{0}\right)^{2} v_{0}}{\left(x_{0}^{2}\left(5 t v_{0}+3 x_{0}\right)^{3}\right)^{4 / 5}}
$$


and its asymptotic approximation

$$
v(t) \sim \frac{1}{5} \frac{3^{2 / 5} \sqrt[5]{125} x_{0}^{2} v_{0}^{3}\left(t^{-1}\right)^{2 / 5}}{\left(v_{0}^{3} x_{0}^{2}\right)^{4 / 5}} .
$$

The velocity as a function of the distance is

$$
v(x)=\frac{x_{0}^{2 / 3} v_{0}}{x^{2 / 3}} .
$$

A first comparison can be made with the laboratory data on turbulent jets of [11] where the velocity of the turbulent jet at the nozzle diameter, $D_{j}=1$, is $v_{0}=2.53 \mathrm{~m} \cdot \mathrm{s}^{-1}$ and at $D_{j}=50$ the centerline velocity is $v=0.314 \mathrm{~m} \cdot \mathrm{s}^{-1}$. The formula (10) with $x_{0}=1$ and $x=50$ gives an averaged velocity of $v=0.186 \mathrm{~m} \cdot \mathrm{s}^{-1}$ which multiplied by 2 gives $v=0.372 \mathrm{~m} \cdot \mathrm{s}^{-1}$. This multiplication by 2 has been done because the turbulent jet develops a profile of velocity in the direction perpendicular to the jet's main axis and therefore the centerline velocity is approximately double that of the averaged velocity. The transit time, $t_{t r}$, necessary to travel a distance of $x_{\max }$ can be derived from Equation (6)

$$
t_{t r}=\frac{3 \sqrt[3]{x_{\max }^{2} x_{0}} x_{\max }-3 x_{0}^{2}}{5 x_{0} v_{0}} .
$$

An astrophysical test can be performed on a typical distance of $15 \mathrm{kpc}$ relative to the jets in 3C 31, see Figure 2 in [12]. On inserting $x=15,000 \mathrm{pc}=15 \mathrm{kpc}, x_{0}=100 \mathrm{pc}$, and $v_{0}=10,000 \mathrm{~km} \cdot \mathrm{s}^{-1}$ we obtain a transit time of $t_{t r}=2.488 \times 10^{7} \mathrm{yr}$.

The rate of mass flow at the point $x, \dot{m}(x)$, is

$$
\dot{m}(x)=\rho v(x) \pi\left(x \tan \left(\frac{\alpha}{2}\right)\right)^{2}
$$

and the astrophysical version is

$$
\dot{m}(x)=0.0237 n x^{4 / 3}(\tan (\alpha / 2))^{2} x_{0}^{2 / 3} \beta_{0} \frac{M_{\odot}}{y r}
$$

where $x$ and $x_{0}$ are expressed in pc, $n$ is the number density of protons expressed in particles $\mathrm{cm}^{-3}, M_{\odot}$ is the solar mass and $\beta_{0}=\frac{v_{0}}{c}$. The previous formula indicates that the rate of transfer of particles is not constant along the jet but increases $\propto x^{4 / 3}$.

\subsection{An Hyperbolic Profile of the Density}

Now the density is assumed to decrease as

$$
\rho=\rho_{0}\left(\frac{x_{0}}{x}\right)
$$

where $\rho_{0}=0$ is the density at $x=x_{0}$. The differential equation that models the energy flux is

$$
x_{0} x(t)\left(\frac{\mathrm{d}}{\mathrm{d} t} x(t)\right)^{3}-v_{0}^{3} x_{0}^{2}=0
$$

and its analytical solution is

$$
x(t)=\frac{1}{3} \sqrt[4]{3} \sqrt[4]{x_{0}\left(4 t v_{0}+3 x_{0}\right)^{3}} .
$$

The asymptotic approximation is 


$$
x(t) \sim \frac{2}{3} \sqrt[4]{3} \sqrt{2} \sqrt[4]{v_{0}^{3} x_{0}} t^{3 / 4}
$$

The analytical solution for the velocity is

$$
v(t)=\frac{\sqrt[4]{3} x_{0}\left(4 t v_{0}+3 x_{0}\right)^{2} v_{0}}{\left(x_{0}\left(4 t v_{0}+3 x_{0}\right)^{3}\right)^{3 / 4}}
$$

and its asymptotic approximation is

$$
v(t) \sim \frac{1}{4} \frac{\sqrt[4]{3} \sqrt[4]{64} x_{0} v_{0}^{3} \sqrt[4]{t^{-1}}}{\left(v_{0}^{3} x_{0}\right)^{3 / 4}} .
$$

The transit time can be derived from Equation (16)

$$
t_{t r}=\frac{3 \sqrt[3]{x_{\max } x_{0}^{2}} x_{\max }-3 x_{0}^{2}}{4 x_{0} v_{0}}
$$

and with $x=15,000 \mathrm{pc}=15 \mathrm{kpc}, \quad x_{0}=100 \mathrm{pc}$, and $v_{0}=10,000 \mathrm{~km} \cdot \mathrm{s}^{-1}$ as in Section 2.1, we have $t_{t r}=5.848 \times 10^{6} \mathrm{yr}$.

\subsection{An Inverse Power Law Profile of the Density}

Here, the density is assumed to decrease as

$$
\rho=\rho_{0}\left(\frac{x_{0}}{x}\right)^{\delta}
$$

where $\rho_{0}$ is the density at $x=x_{0}$. The differential equation which models the energy flux is

$$
\frac{1}{2}\left(\frac{x_{0}}{x}\right)^{\delta}\left(\frac{\mathrm{d}}{\mathrm{d} t} x(t)\right)^{2} x^{2}-\frac{1}{2} v_{0}^{2} x_{0}^{2}=0
$$

There is no analytical solution, and we simply express the velocity as a function of the position, $x$,

$$
v(x)=\frac{x_{0} v_{0}}{x} \frac{1}{\sqrt{\left(\frac{x_{0}}{x}\right)^{\delta}}}
$$

see Figure 1

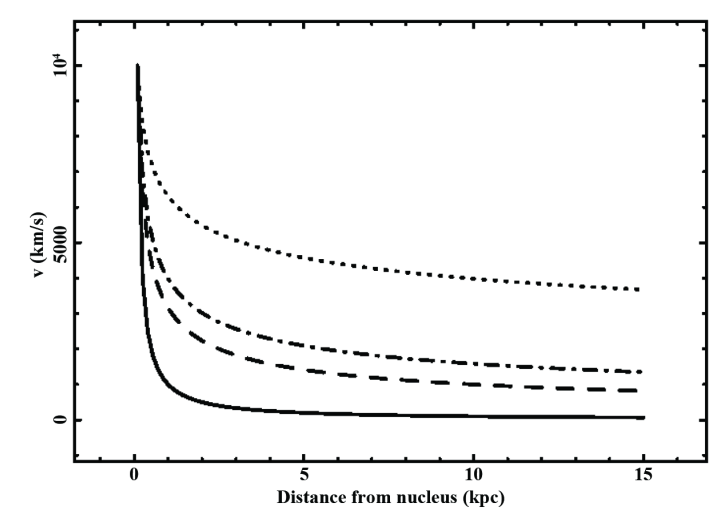

Figure 1. Classical velocity as a function of the distance from the nucleus when $x_{0}=100 \mathrm{pc}$ and $v_{0}=10,000 \mathrm{~km} \cdot \mathrm{s}^{-1}: \delta=0$ (full line), $\delta=1$ (dashes), $\delta=1.2$ (dot-dash-dot-dash) and $\delta=1.6$ (dotted). 
The rate of mass flow at the point $x$ is

$$
\dot{m}(x)=\rho_{0} \sqrt{\left(\frac{x_{0}}{x}\right)^{\delta}} \pi x(\tan (\alpha / 2))^{2} x_{0} v_{0}
$$

and the astrophysical version is

$$
\dot{m}(x)=0.0237 n_{0} \sqrt{\left(1.0 \frac{x_{0}}{x}\right)^{\delta}} x(\tan (\alpha / 2))^{2} x_{0} \beta_{0} \frac{M_{\odot}}{y r}
$$

where $n_{0}$ is the number density of protons expressed in particles $\mathrm{cm}^{-3}$ at $x_{0}$. The previous formula indicates that the rate of transfer of particles scales $\propto x^{1-\frac{1}{2} \delta}$ and therefore at $\delta=2$ is constant.

\section{Relativistic Turbulent Jets}

The conservation of the energy flux in special relativity (SR) in the presence of a velocity $v$ along one direction states that

$$
A(x) \frac{1}{1-\frac{v^{2}}{c^{2}}}\left(e_{0}+p_{0}\right) v=\text { cost }
$$

where $A(x)$ is the considered area in the direction perpendicular to the motion, $c$ is the speed of light, $e_{0}=c^{2} \rho$ is the energy density in the rest frame of the moving fluid, and $p_{0}$ is the pressure in the rest frame of the moving fluid, see formula A31 in [10]. In accordance with the current models of classical turbulent jets, we insert $p_{0}=0$ and the conservation law for relativistic energy flux is

$$
\rho c^{2} v \frac{1}{1-\frac{v^{2}}{c^{2}}} A(x)=\text { cost. }
$$

Our physical units are pc for length and yr for time, and in these units, the speed of light is $c=0.306 \mathrm{pc} \cdot \mathrm{yr}^{-1}$. A discussion of the mass-energy equivalence principle in fluids can be found in [13].

\subsection{Constant Density in SR}

The conservation of the relativistic energy flux when the density is constant can be written as a differential equation

$$
\begin{aligned}
& \rho c^{2}\left(\frac{\mathrm{d}}{\mathrm{d} t} x(t)\right) \pi(x(t))^{2}\left(\tan \left(\frac{\alpha}{2}\right)\right)^{2}\left(1-\frac{\left(\frac{\mathrm{d}}{\mathrm{d} t} x(t)\right)^{2}}{c^{2}}\right)^{-1} \\
& -\rho c^{2} v_{0} \pi x_{0}^{2}\left(\tan \left(\frac{\alpha}{2}\right)\right)^{2}\left(1-\frac{v_{0}^{2}}{c^{2}}\right)^{-1}=0 .
\end{aligned}
$$

An analytical solution of the previous differential equation at the moment of writing does not exist but we can provide a power series solution of the form

$$
x(t)=a_{0}+a_{1} t+a_{2} t^{2}+a_{3} t^{3}+\cdots
$$

see [14] [15]. The coefficients $a_{n}$ up to order 4 are 


$$
\begin{aligned}
& a_{0}=x_{0} \\
& a_{1}=v_{0} \\
& a_{2}=\frac{1}{3} \frac{v_{0}^{3}\left(5 c^{6}-11 c^{4} v_{0}^{2}+3 c^{2} v_{0}^{4}+3 v_{0}^{6}\right)}{x_{0}^{2}\left(c^{2}+v_{0}^{2}\right)\left(c^{4}+2 c^{2} v_{0}^{2}+v_{0}^{4}\right)} \\
& a_{3}=\frac{1}{3} \frac{v_{0}^{3}\left(5 c^{6}-11 c^{4} v_{0}^{2}+3 c^{2} v_{0}^{4}+3 v_{0}^{6}\right)}{x_{0}^{2}\left(c^{2}+v_{0}^{2}\right)\left(c^{4}+2 c^{2} v_{0}^{2}+v_{0}^{4}\right)} .
\end{aligned}
$$
(28)

In order to find a numerical solution of the above differential equation we isolate the velocity from Equation

$$
v\left(x ; x_{0}, \beta_{0}, c\right)=\frac{1}{2} \frac{\left(\beta_{0}^{2} x^{2}-x^{2}+\sqrt{x^{4} \beta_{0}^{4}-2 x^{4} \beta_{0}^{2}+4 \beta_{0}^{2} x_{0}^{4}+x^{4}}\right) c}{\beta_{0} x_{0}^{2}}
$$

where $\beta_{0}=\frac{v_{0}}{c}$ and separate the variables

$$
\int_{x_{0}}^{x} 2 \frac{\beta_{0} x_{0}^{2}}{\left(\beta_{0}^{2} x^{2}-x^{2}+\sqrt{x^{4} \beta_{0}^{4}-2 x^{4} \beta_{0}^{2}+4 \beta_{0}^{2} x_{0}^{4}+x^{4}}\right) c} \mathrm{~d} x=\int_{0}^{t} \mathrm{~d} t .
$$

The indefinite integral on the left side of the previous equation has an analytical expression

$$
I\left(x ; \beta_{0}, c, x_{0}\right)=\frac{A N}{A D}
$$

where

$$
\begin{aligned}
& A N=2 \beta_{0}^{3} x_{0}^{6} \sqrt{2} \sqrt{4-2 \frac{i \beta_{0} x^{2}}{x_{0}^{2}}+2 \frac{i x^{2}}{\beta_{0} x_{0}^{2}}} \sqrt{4+2 \frac{i \beta_{0} x^{2}}{x_{0}^{2}}-2 \frac{i x^{2}}{\beta_{0} x_{0}^{2}}} \times F\left(1 / 2 x \sqrt{2} \sqrt{\frac{i\left(\beta_{0}^{2}-1\right)}{\beta_{0} x_{0}^{2}}}, i\right) \\
& -\beta_{0}^{3} x_{0}^{2} x^{3} \sqrt{\frac{i \beta_{0}}{x_{0}^{2}}-\frac{i}{\beta_{0} x_{0}^{2}}} \sqrt{x^{4} \beta_{0}^{4}-2 x^{4} \beta_{0}^{2}+4 \beta_{0}^{2} x_{0}^{4}+x^{4}} \\
& +\beta_{0} x_{0}^{2} x^{3} \sqrt{\frac{i \beta_{0}}{x_{0}^{2}}-\frac{i}{\beta_{0} x_{0}^{2}}} \sqrt{x^{4} \beta_{0}^{4}-2 x^{4} \beta_{0}^{2}+4 \beta_{0}^{2} x_{0}^{4}+x^{4}} \\
& +\beta_{0}^{5} x_{0}^{2} x^{5} \sqrt{\frac{i \beta_{0}}{x_{0}^{2}}-\frac{i}{\beta_{0} x_{0}^{2}}}-2 \beta_{0}^{3} x_{0}^{2} x^{5} \sqrt{\frac{i \beta_{0}}{x_{0}^{2}}-\frac{i}{\beta_{0} x_{0}^{2}}} \\
& +4 \beta_{0}^{3} x_{0}^{6} x \sqrt{\frac{i \beta_{0}}{x_{0}^{2}}-\frac{i}{\beta_{0} x_{0}^{2}}}+\beta_{0} x_{0}^{2} x^{5} \sqrt{\frac{i \beta_{0}}{x_{0}^{2}}-\frac{i}{\beta_{0} x_{0}^{2}}}
\end{aligned}
$$

and

$$
A D=6 c \beta_{0}^{2} x_{0}^{4} \sqrt{\frac{i \beta_{0}}{x_{0}^{2}}-\frac{i}{\beta_{0} x_{0}^{2}}} \sqrt{x^{4} \beta_{0}^{4}-2 x^{4} \beta_{0}^{2}+4 \beta_{0}^{2} x_{0}^{4}+x^{4}}
$$

where $i=\sqrt{-1}$ and

$$
F(x ; m)=\int_{0}^{x} \frac{1}{\sqrt{1-t^{2}} \sqrt{1-m^{2} t^{2}}} \mathrm{~d} t
$$

is the elliptic integral of the first kind, see formula 17.2.7 in [16]. Figure 2 shows the behaviour of $\beta$ as 


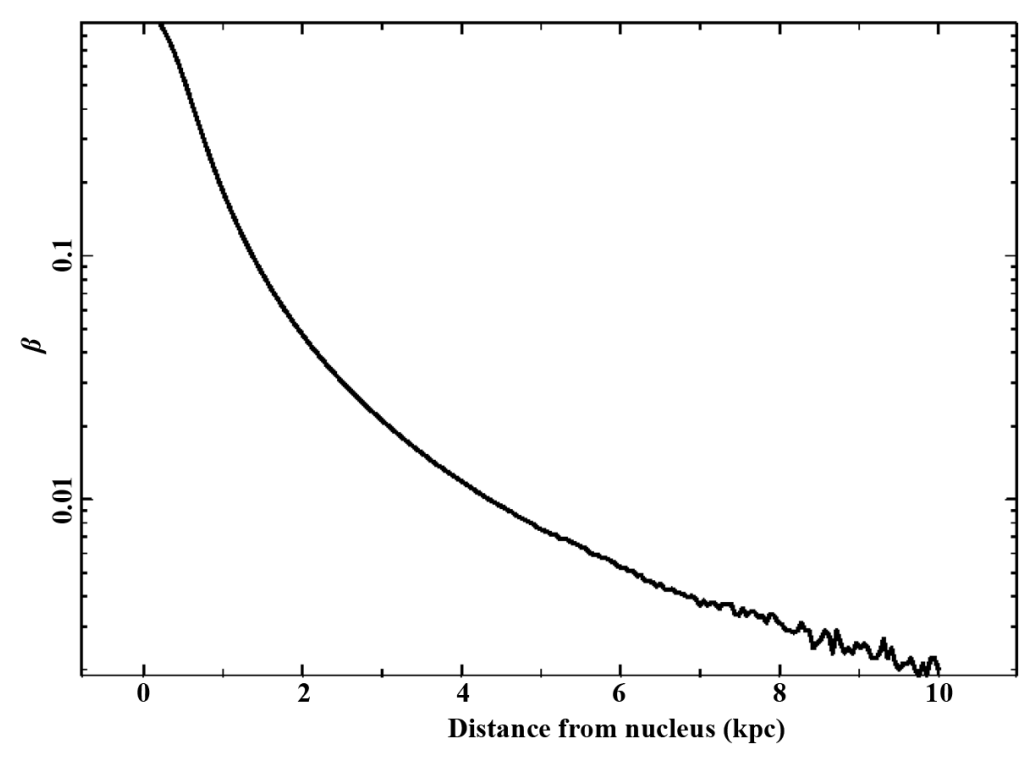

Figure 2. Relativistic $\beta$ as a function of the distance from the nucleus when $x_{0}=200 \mathrm{pc}$ and $\beta_{0}=0.9$ in the case of constant density.

function of the distance.

A numerical solution can be found by solving the following non-linear equation

$$
I\left(x ; \beta_{0}, c, x_{0}\right)-I\left(x_{0} ; \beta_{0}, c, x_{0}\right)=t
$$

and Figure 3 presents a typical comparison with the series solution.

The relativistic rate of mass flow in the case of constant density is

$$
\dot{m}(x)=\frac{\rho\left(\beta_{0}^{2} x^{2}-x^{2}+\sqrt{x^{4} \beta_{0}^{4}-2 x^{4} \beta_{0}^{2}+4 \beta_{0}^{2} x_{0}^{4}+x^{4}}\right) c \pi x(\tan (\alpha / 2))^{2}}{\sqrt{2\left(1-\beta_{0}^{2}\right)\left(\beta_{0}^{2} x^{2}-x^{2}+\sqrt{x^{4} \beta_{0}^{4}-2 x^{4} \beta_{0}^{2}+4 \beta_{0}^{2} x_{0}^{4}+x^{4}}\right)}}
$$

\subsection{Inverse Power Law Profile of Density in SR}

The conservation of the relativistic energy flux in the presence of an inverse power law density profile as given by Equation (21) is

$$
\begin{aligned}
& \rho_{0} c^{2}\left(\frac{\mathrm{d}}{\mathrm{d} t} x(t)\right) \pi(x(t))^{2}\left(\tan \left(\frac{\alpha}{2}\right)\right)^{2}\left(\frac{x_{0}}{x(t)}\right)^{\delta}\left(-\frac{\left(\frac{\mathrm{d}}{\mathrm{d} t} x(t)\right)^{2}}{c^{2}}+1\right)^{-1} \\
& -\rho_{0} c^{2} v_{0} \pi x_{0}^{2}\left(\tan \left(\frac{\alpha}{2}\right)\right)^{2}\left(-\frac{v_{0}^{2}}{c^{2}}+1\right)^{-1}=0 .
\end{aligned}
$$

This differential equation does not have an analytical solution. An expression for $\beta$ as a function of the distance is

$$
\beta(x)=\frac{1}{2} \frac{1}{\beta_{0} x_{0}^{2}}\left(\beta_{0}^{2} x^{2}\left(\frac{x_{0}}{x}\right)^{\delta}-x^{2}\left(\frac{x_{0}}{x}\right)^{\delta}+\sqrt{D}\right)
$$

with 


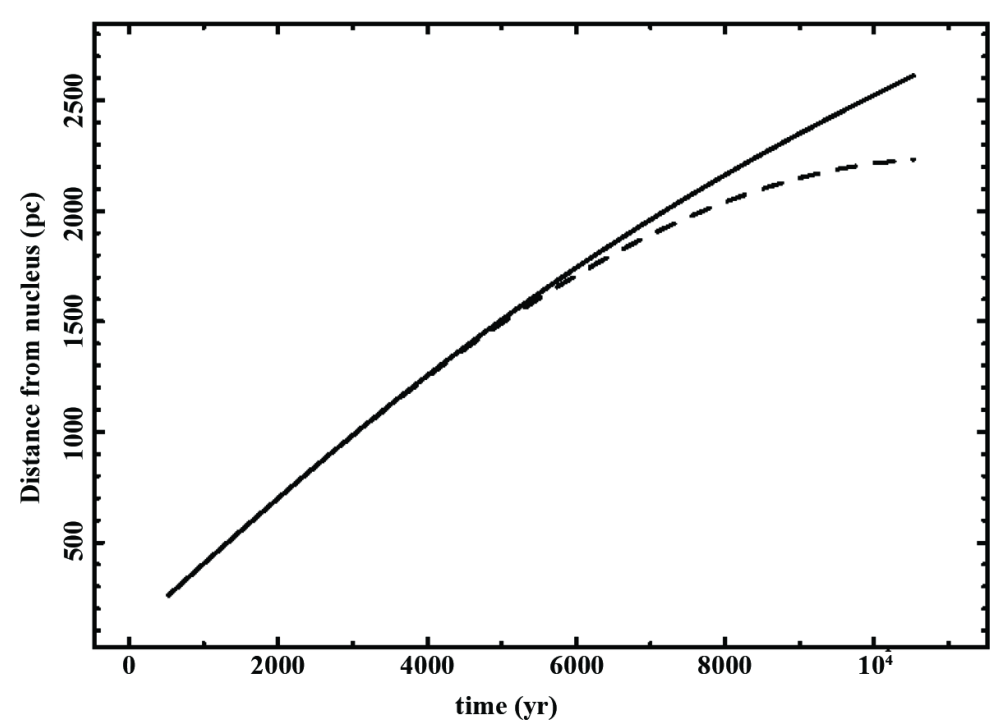

Figure 3. Non-linear relativistic solution as given by Equation (37) (full line) and series solution as given by Equation (29) (dashed line) when $x_{0}=100 \mathrm{pc}$ and $\beta_{0}=0.999$.

$$
D=\left(\left(\frac{x_{0}}{x}\right)^{\delta}\right)^{2} \beta_{0}^{4} x^{4}-2\left(\left(\frac{x_{0}}{x}\right)^{\delta}\right)^{2} \beta_{0}^{2} x^{4}+\left(\left(\frac{x_{0}}{x}\right)^{\delta}\right)^{2} x^{4}+4 \beta_{0}^{2} x_{0}^{4} .
$$

The behaviour of $\beta$ as a function of the distance for different values of $\delta$ can be seen in Figure 4. A power series solution for the above differential equation (39) up to order three gives

$$
\begin{aligned}
& a_{0}=x_{0} \\
& a_{1}=v_{0} \\
& a_{2}=\frac{1}{2} \frac{v_{0}^{2}\left(c^{2} \delta-\delta v_{0}^{2}-2 c^{2}+2 v_{0}^{2}\right)}{x_{0}\left(c^{2}+v_{0}^{2}\right)} .
\end{aligned}
$$

Figure 5 shows a comparison between the numerical solution of (39) with the series solution.

Non-linear relativistic solution as given by Equation (39) (full line) and series solution as given by Equation (42) (dashed line) when $x_{0}=100 \mathrm{pc}$ and $\beta_{0}=0.999$.

The relativistic rate of mass flow in the case of an inverse power law for the density is

$$
\dot{m}(x)=\frac{\rho_{0}\left(\frac{x_{0}}{x}\right)^{\delta}\left(\beta_{0}^{2} x^{2}\left(\frac{x_{0}}{x}\right)^{\delta}-x^{2}\left(\frac{x_{0}}{x}\right)^{\delta}+\sqrt{D}\right) c \pi x^{2}(\tan (\alpha / 2))^{2}}{2 \beta_{0} x_{0}^{2} \sqrt{-1 / 4 \frac{1}{\beta_{0}^{2} x_{0}^{4}}\left(\beta_{0}^{2} x^{2}\left(\frac{x_{0}}{x}\right)^{\delta}-x^{2}\left(\frac{x_{0}}{x}\right)^{\delta}+\sqrt{D}\right)^{2}+1}}
$$

where $\rho_{0}$ is the density at $x_{0}$ and $D$ was defined in Equation (41).

\section{The Losses}

The previous analysis does not cover the radiative losses. The astrophysical version of the relativistic energy flux as represented by Equation (27) is

$$
\frac{\mathrm{d} E}{\mathrm{~d} t}=1.348 \times 10^{49} \frac{n \beta_{0} R_{100}^{2}}{1-\beta_{0}^{2}} \frac{\mathrm{erg}}{\mathrm{s}}
$$




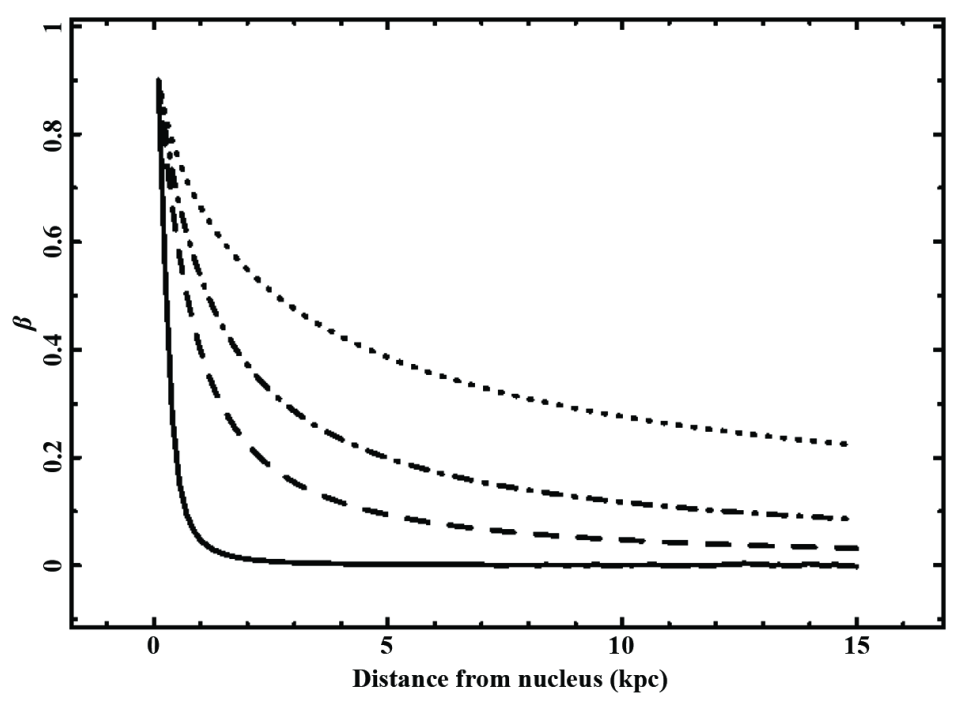

Figure 4. Relativistic $\beta$ for the relativistic energy flux conservation as a function of the distance from the nucleus when $x_{0}=100 \mathrm{pc}$ and $\beta_{0}=0.9$ : $\delta=0$ (full line), $\delta=1$ (dashes), $\delta=1.2$ (dot-dash-dot-dash) and $\delta=1.4$ (dotted).

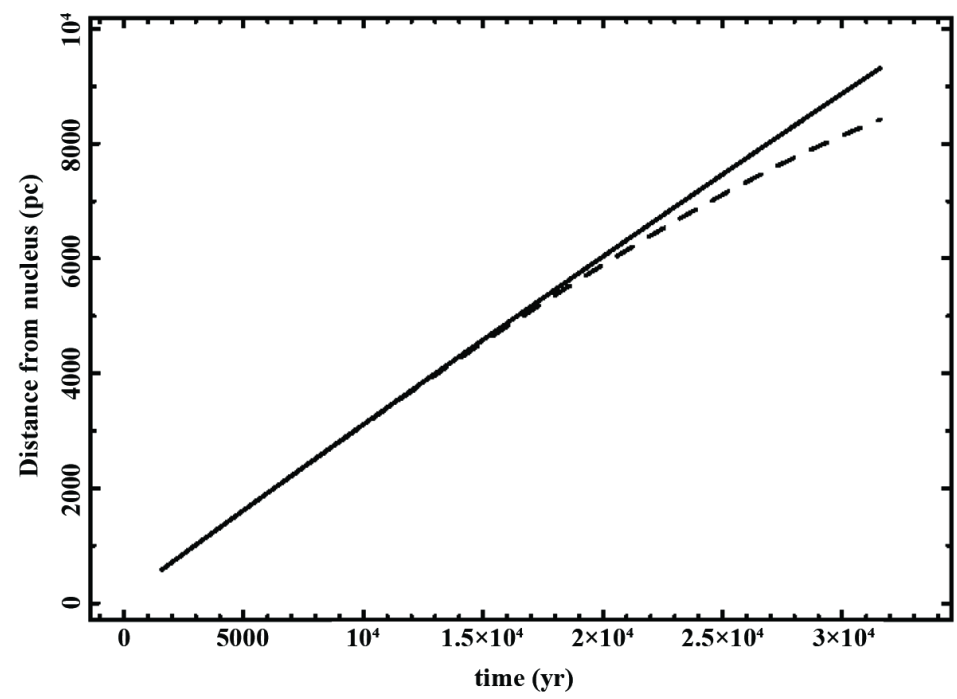

Figure 5. Non-linear relativistic solution as given by Equation (39) (full line) and series solution as given by Equation (42) (dashed line) when $x_{0}=100 \mathrm{pc}$ and $\beta_{0}=0.999$.

where $R_{100}$ is the radius of the jet expressed in units of $100 \mathrm{pc}$, and $n$ is the number density of protons expressed in particles $\mathrm{cm}^{-3}$. The above luminosity is $4-5$ orders of magnitude too high for the radio sources here considered. In order to explain this discrepancy, one model assumes that extragalactic jets are much lighter than the surroundings. The second model assumes that the observed intensity of radiation, $I_{v}$, at a given frequency $v$ is a fraction of the energy flux

$$
I_{v}=\varepsilon \frac{\mathrm{d} E}{\mathrm{~d} t} \frac{\mathrm{erg}}{\mathrm{s}}
$$

where $\varepsilon$ represents the efficiency of conversion of the relativistic energy flux into radiation. At the moment of writing there is no exact evaluation of the efficiency of conversion. We now outline two different models for the 
radiative losses and a model for the magnetic field.

\subsection{Losses through Recursion}

In the classical case, with constant density, we can model the radiative losses through the following recursive equation obtained by modifying Equation (5)

$$
\frac{1}{2} \rho v_{n+1}^{3} \pi\left(x_{n+1} \tan \left(\frac{\alpha}{2}\right)\right)^{2}+\varepsilon \frac{1}{2} \rho v_{n}^{3} \pi\left(x_{n} \tan \left(\frac{\alpha}{2}\right)\right)^{2}=\frac{1}{2} \rho v_{n}^{3} \pi\left(x_{n} \tan \left(\frac{\alpha}{2}\right)\right)^{2}
$$

where

$$
x_{n+1}=x_{n}+v_{n} \Delta t .
$$

Here $n$ starts from $0, v_{n}$ is the velocity at the $n$th step, $x_{n}$ is the position at the $n$th step, $\varepsilon$ is the efficiency of conversion into radiation, $\alpha$ is the jet's opening angle, and $\Delta t$ is the temporal step. The velocity at step $n+1$ is

$$
v_{n+1}=\frac{x_{n}^{2 / 3} \sqrt[3]{1-\varepsilon} v_{n}}{\left(v_{n} \Delta t+x_{n}\right)^{2 / 3}} .
$$

Figure 6 shows the velocity as a function of the distance; $\varepsilon \approx 10^{-4}$ does not modify in an appreciable way the velocity.

In the relativistic case, with constant density, the radiative losses are modeled by a modification of Eq. (28) and the following recursive equation for the velocity at step $n+1$ is obtained

$$
v_{n+1}=\frac{N_{n}}{D_{n}}
$$

where

$$
\begin{aligned}
N_{n}= & c^{4} \Delta^{2} t^{2} v_{n}^{2}-c^{2} \Delta^{2} t^{2} v_{n}^{4}+2 c^{4} \Delta t v_{n} x_{n}-2 c^{2} \Delta t v_{n}^{3} x_{n} \\
& +c^{4} x_{n}^{2}-c^{2} v_{n}^{2} x_{n}^{2}-c^{4} \sqrt{S_{n}} \\
S_{n}= & \frac{v_{n}^{4}\left(v_{n} \Delta t+x_{n}\right)^{4}}{c^{4}}+4 \frac{v_{n}^{2} \varepsilon^{2} x_{n}^{4}}{c^{2}}-8 \frac{v_{n}^{2} \varepsilon x_{n}^{4}}{c^{2}}-2 \frac{v_{n}^{2}\left(v_{n} \Delta t+x_{n}\right)^{4}}{c^{2}} \\
& +4 \frac{v_{n}^{2} x_{n}^{4}}{c^{2}}+\left(v_{n} \Delta t+x_{n}\right)^{4} \\
D_{n}= & 2 c^{2} v_{n} x_{n}^{2} \varepsilon-2 c^{2} v_{n} x_{n}^{2} .
\end{aligned}
$$

Figure 7 shows the relativistic velocity as a function of the distance and $\varepsilon$.

\subsection{The Parametrization of the Losses}

The radiative losses can also be modeled by an "ad hoc" law for the available flux of kinetic energy, which is assumed to decrease with an inverse power law of the type $\propto\left(\frac{x_{0}}{x}\right)^{\eta}$. The resulting differential equation in SR with constant density is

$$
\begin{aligned}
& \rho c^{2}\left(\frac{\mathrm{d}}{\mathrm{d} t} x(t)\right) \pi(x(t))^{2}\left(\tan \left(\frac{\alpha}{2}\right)\right)^{2}\left(1-\frac{\left(\frac{\mathrm{d}}{\mathrm{d} t} x(t)\right)^{2}}{c^{2}}\right)^{-1} \\
& -\rho c^{2} v_{0} \pi x_{0}^{2}\left(\tan \left(\frac{\alpha}{2}\right)\right)^{2}\left(1-\frac{v_{0}^{2}}{c^{2}}\right)^{-1}\left(\frac{x_{0}}{x}\right)^{\eta}=0 .
\end{aligned}
$$




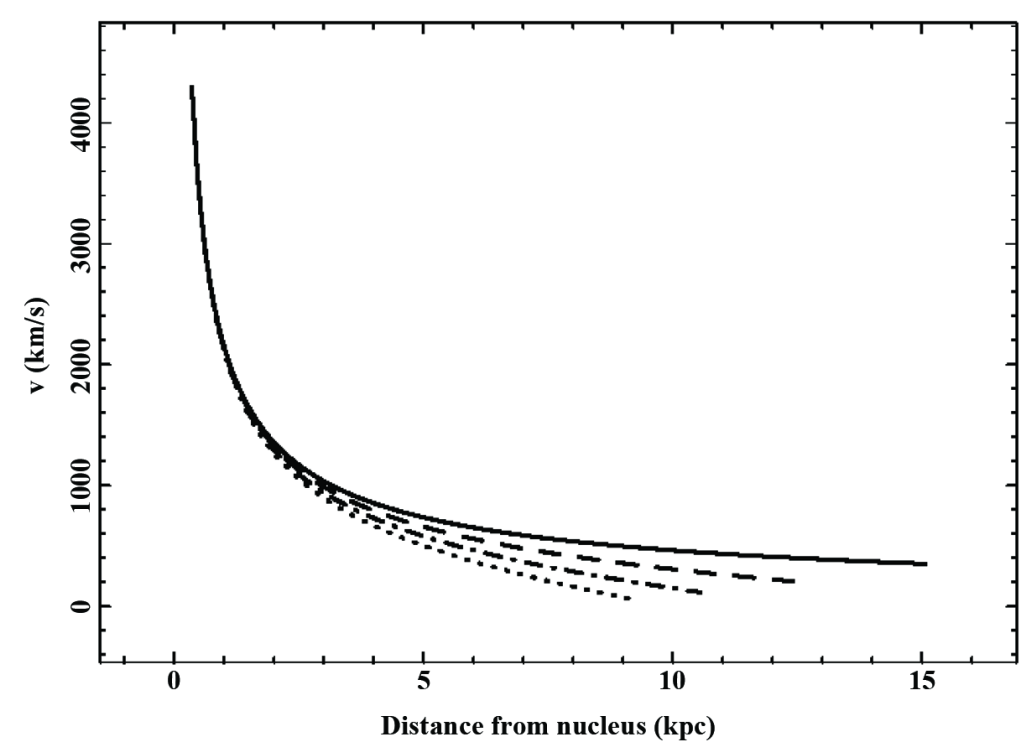

Figure 6. Classical velocity as a function of the distance from the nucleus when $x_{0}=100 \mathrm{pc}, \Delta t=2.5 \times 10^{4} \mathrm{yr}$ and $v_{0}=10,000 \mathrm{~km} \cdot \mathrm{s}^{-1}: \varepsilon=0$ (full line), $\varepsilon=0.002$ (dashes), $\varepsilon=0.004$ (dot-dash-dot-dash) and $\varepsilon=0.006$ (dotted).

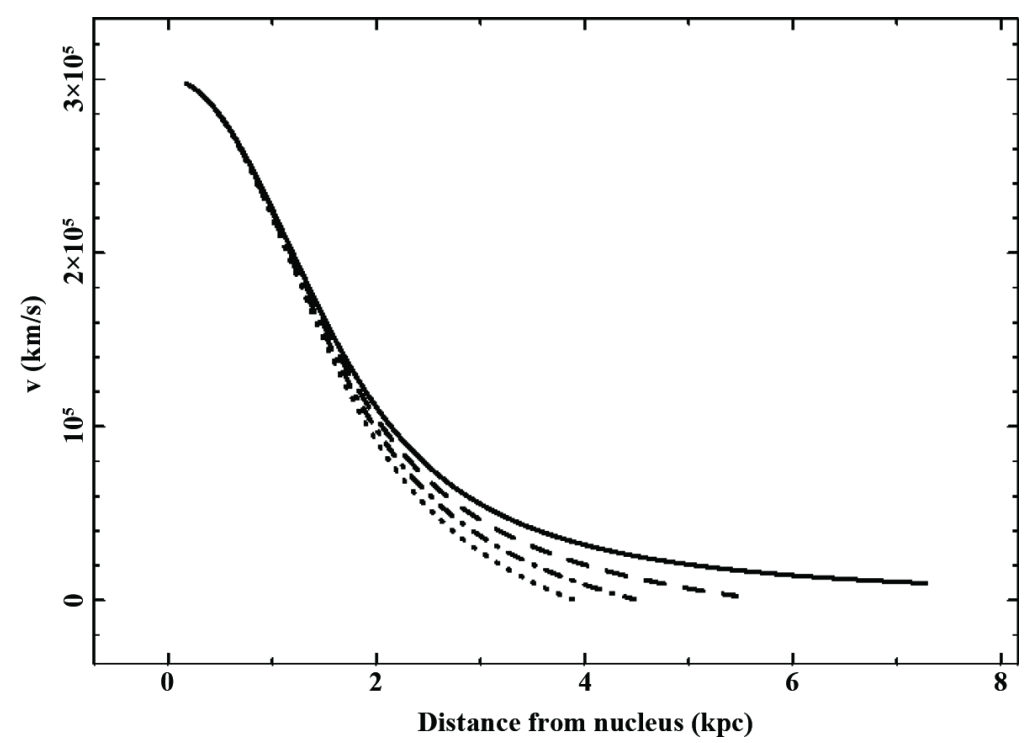

Figure 7. Relativistic velocity as a function of the distance from the nucleus when $x_{0}=100 \mathrm{pc}, \Delta t=250 \mathrm{yr}$, and $\beta_{0}=0.999: \varepsilon=0$ (full line), $\varepsilon=0.002$ (dashes), $\varepsilon=0.004$ (dot-dash-dot-dash) and $\varepsilon=0.006$ (dotted).

Figure 8 shows the numerical trajectory as a function of time for different values of the exponent $\eta$ : an increase in $\eta$ means a lower value for the traveled distance.

\subsection{The Magnetic Field}

The magnetic field in CGS has an energy density of $\frac{B^{2}}{8 \pi}$ where $B$ is the magnetic field. The presence of the magnetic field can be modeled by adding a second term for the density of energy in the rest frame of the moving fluid, see Equation (39) which models the relativistic flow of energy the in presence of an inverse power law 


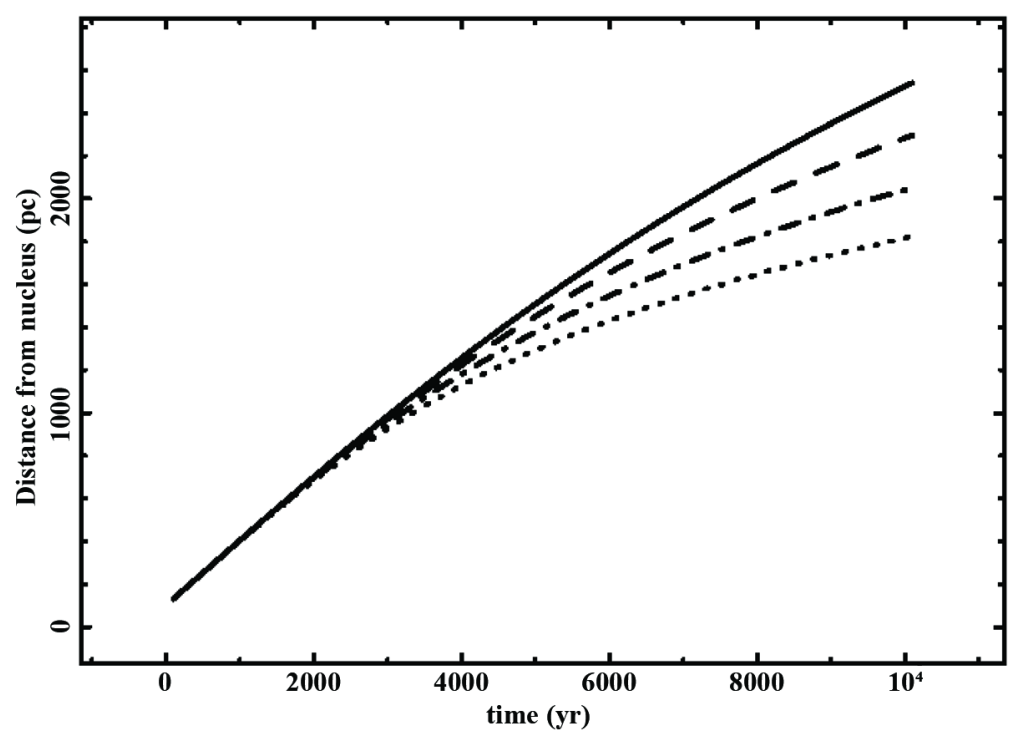

Figure 8. Relativistic distance as a function of time when $x_{0}=100 \mathrm{pc}$, and $\beta_{0}=0.999: \eta=0$ (full line), $\eta=0.2$ (dashes), $\eta=0.4 \quad$ (dot-dash-dotdash) and $\eta=0.6$ (dotted).

$$
\begin{aligned}
& \left(\rho_{0} c^{2}+\frac{B^{2}}{8 \pi}\right)\left(\frac{\mathrm{d}}{\mathrm{d} t} x(t)\right) \pi(x(t))^{2}\left(\tan \left(\frac{\alpha}{2}\right)\right)^{2}\left(\frac{x_{0}}{x(t)}\right)^{\delta}\left(-\frac{\left(\frac{\mathrm{d}}{\mathrm{d} t} x(t)\right)^{2}}{c^{2}}+1\right)^{-1} \\
& -\left(\rho_{0} c^{2}+\frac{B_{0}^{2}}{8 \pi}\right) v_{0} \pi x_{0}^{2}\left(\tan \left(\frac{\alpha}{2}\right)\right)^{2}\left(-\frac{v_{0}^{2}}{c^{2}}+1\right)^{-1}=0 .
\end{aligned}
$$

We continue assuming a constant of proportionality between the density of energy of the magnetic field and the rest mass all along the jet

$$
\frac{B(x)^{2}}{8 \pi} \propto \rho c^{2} \propto\left(\frac{x_{0}}{x}\right)^{\delta} .
$$

The magnetic field as a function of the distance $x$ is

$$
B=\sqrt{B_{0}^{2}\left(\frac{x_{0}}{x}\right)^{\delta}}
$$

where $B_{0}$ is the magnetic field at $x=x_{0}$. We assume an inverse power law spectrum for the ultrarelativistic electrons of the type

$$
N(E) \mathrm{d} E=K E^{-p} \mathrm{~d} E
$$

where $K$ is a constant and $p$ the exponent of the inverse power law. The intensity of the synchrotron radiation has a standard expression, as given by formula (1.175) in [17],

$$
I(v) \approx 0.933 \times 10^{-23} \alpha_{p}(p) K l H_{\perp}^{(p+1) / 2}\left(\frac{6.26 \times 10^{18}}{v}\right)^{(p-1) / 2} \mathrm{erg} \cdot \mathrm{sec}^{-1} \cdot \mathrm{cm}^{-2} \cdot \mathrm{Hz}^{-1} \cdot \mathrm{rad}^{-2}
$$

where $v$ is the frequency, $H_{\perp}$ is the magnetic field perpendicular to the electron's velocity, $l$ is the dimension 
of the radiating region along the line of sight, and $\alpha_{p}(p)$ is a slowly varying function of $p$ which is of the order of unity. As an example, $p=2.5$ produces an intensity of the type $I(v) \propto v^{-0.75}$.

We now analyse the intensity along the centerline of the jet, which means constant radiating length. The intensity, assuming a constant $p$, scales as

$$
I(x)=\frac{I_{0} B(x)^{p / 2+1 / 2}}{B_{0}^{p / 2+1 / 2}}
$$

where $I_{0}$ is the intensity at $x=x_{0}$ and $B_{0}$ the magnetic field at $x=x_{0}$. We insert Equation (53) in order to have an analytical expression for the centerline intensity

$$
I(x)=B_{0}^{-p / 2-1 / 2} I_{0}\left(B_{0}^{2}\left(\frac{x_{0}}{x}\right)^{\delta}\right)^{p / 4+1 / 4}
$$

and Figure 9 shows the theoretical synchrotron intensity as well the observed one in 3C31, see Figure 8 in [12]. We test the goodness of fit through two standard statistical tests. The first test is the $\chi^{2}$, which is computed as

$$
\chi^{2}=\sum_{j=1}^{n}\left(I_{o b s}-I_{\text {theo }}\right)^{2}
$$

where the index $j$ varies from 1 to the number of available observations, $n, I_{o b s}$ is the observed intensity at position $j$, and $I_{\text {theo }}$ is the observed one. A second test of the model works over different points of the jet and an observational percentage of reliability, $\epsilon_{o b s}$, is introduced

$$
\epsilon_{\text {obs }}=100\left(1-\frac{\sum_{j}\left|I_{o b s}-I_{\text {theo }}\right|_{j}}{\sum_{j} I_{\text {theo }, j}}\right) .
$$

Another application is to the spatial evolution of the magnetic field of 3C273 as observed by VLBA in the pc region, see [18]. Figure 10 shows the observed behaviour of the magnetic field as well the theoretical evolution as represented by Equation (53).

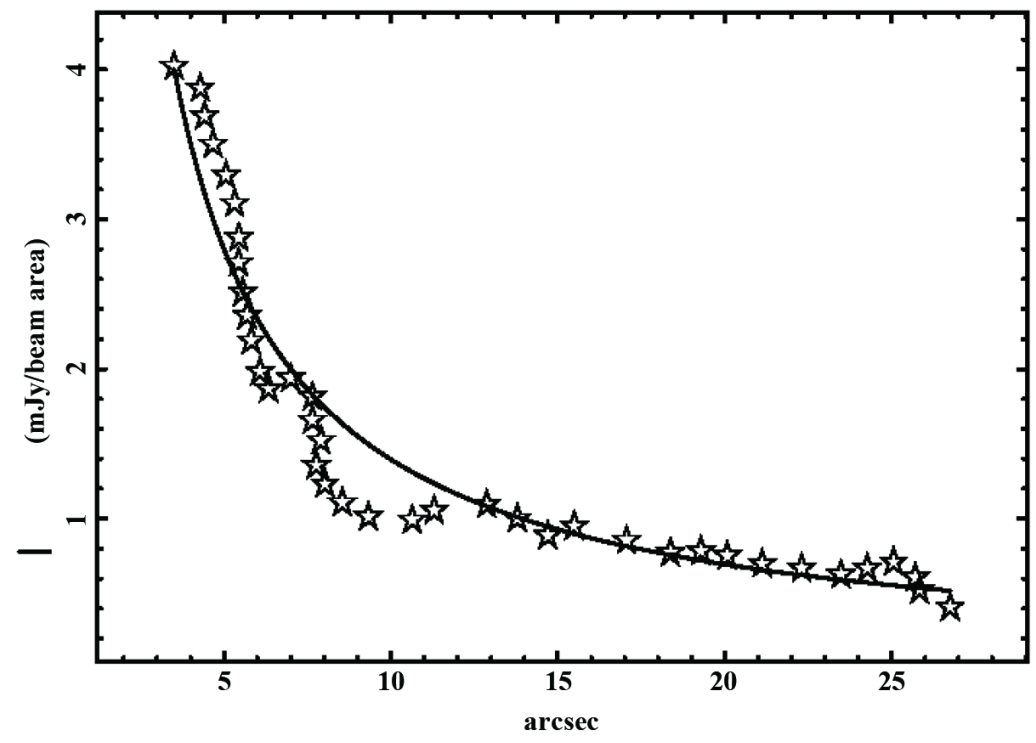

Figure 9. Intensity profile along the centerline of 3C31 when $x_{0}=3.51 \mathrm{arcsec}$, $I_{0}=4 \mathrm{mJy} /\left(\right.$ beam area), $p=2.5, B_{0}=10^{-4}$ gauss, $\delta=1.15, \epsilon_{o b s}=87.56 \%$ and $\chi^{2}=3.05$. 


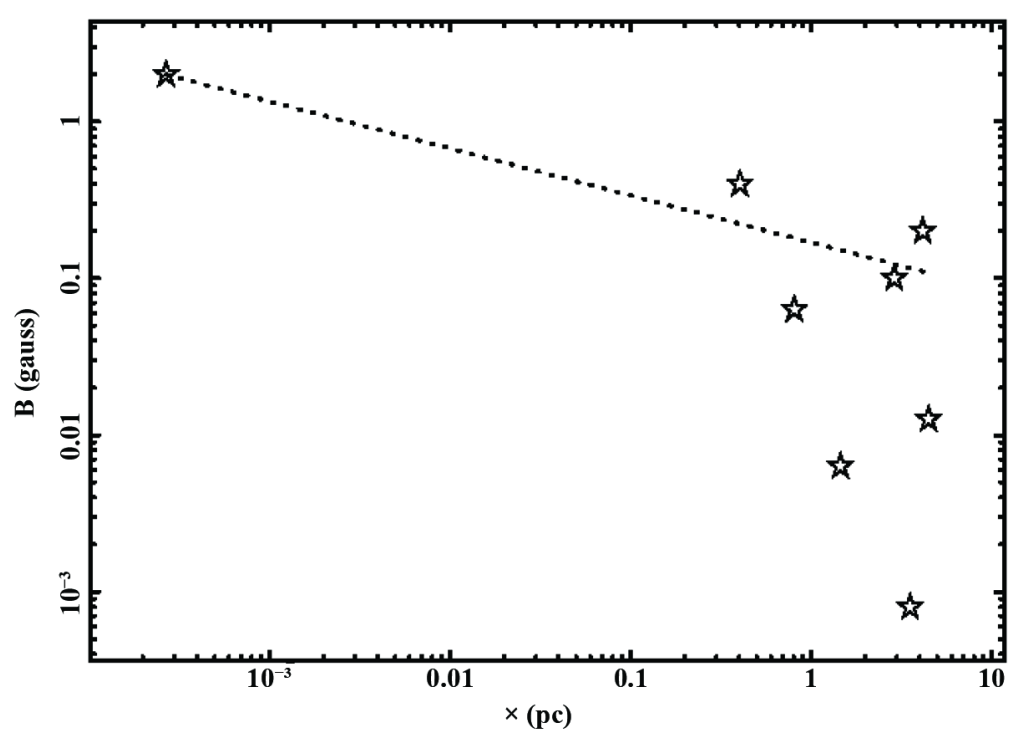

Figure 10. Observed magnetic field density of 3C273 as a function of the distance, empty stars, and theoretical curve as represented by Equation (53), dotted line, when $x_{0}=2.6 \times 10^{-4} \mathrm{pc}, B_{0}=2$ gauss, $\delta=0.6$.

The analytical expression for the magnetic field as a function of the distance allows finding the maximum energy which can be reached in the process of acceleration of the cosmic rays in extragalactic radio-sources. The Hillas argument, see [19], firstly introduces the relativistic ions' gyro-radius, $\rho_{Z}$, expressing the energy in $10^{15}$ $\mathrm{eV}$ units $\left(E_{15}\right)$, the magnetic field in $10^{-6}$ gauss $\left(B_{-6}\right)$

$$
\rho_{Z}=1.08 \frac{E_{15}}{B_{-6} Z} \mathrm{pc}
$$

where $Z$ is the atomic number. The relativistic gyro-radius is equalized to the maximum transversal dimension of the jet, which is the diameter,

$$
\rho_{Z}=2 x \tan \left(\frac{\alpha}{2}\right)
$$

The resulting expression for the maximum energy is

$$
E_{15}=9.25 \times 10^{5} x \tan \left(\frac{\alpha}{2}\right) \sqrt{B_{0}^{2}\left(\frac{x_{0}}{x}\right)^{\delta}} Z
$$

where $B_{0}$ is expressed in gauss and $x$ and $x_{0}$ in pc. Figure 11 reports the Hillas plot for 3C31 from which it is possible to say that $E_{15}=10^{6}$ or $E=10^{21} \mathrm{eV}$ can be reached at the end of the jet when the magnetic field at $x_{0}=100 \mathrm{pc}$ is $B_{0}=0.025$ gauss.

\section{Conclusions}

Classical turbulence: We modeled the physics of turbulent jets by the conservation of the energy flux. In the case of constant density, we derived solutions for the distance and velocity as functions of time, see Equation (6) and Equation (8). In the presence of an hyperbolic profile of density, the solutions for the distance and velocity as functions of time are Equation (16) and Equation (18). The case of a density which follows an inverse power law of density is limited to the derivation of the velocity, see Equation (23). The presence of an inverse power law introduces flexibility in the results and as an example when $\delta=2$ the rate of mass flow does not increase with $x$ but is constant, see Equation (24).

Relativistic turbulence: The conservation of the relativistic energy flux for turbulent jets is here analysed in 


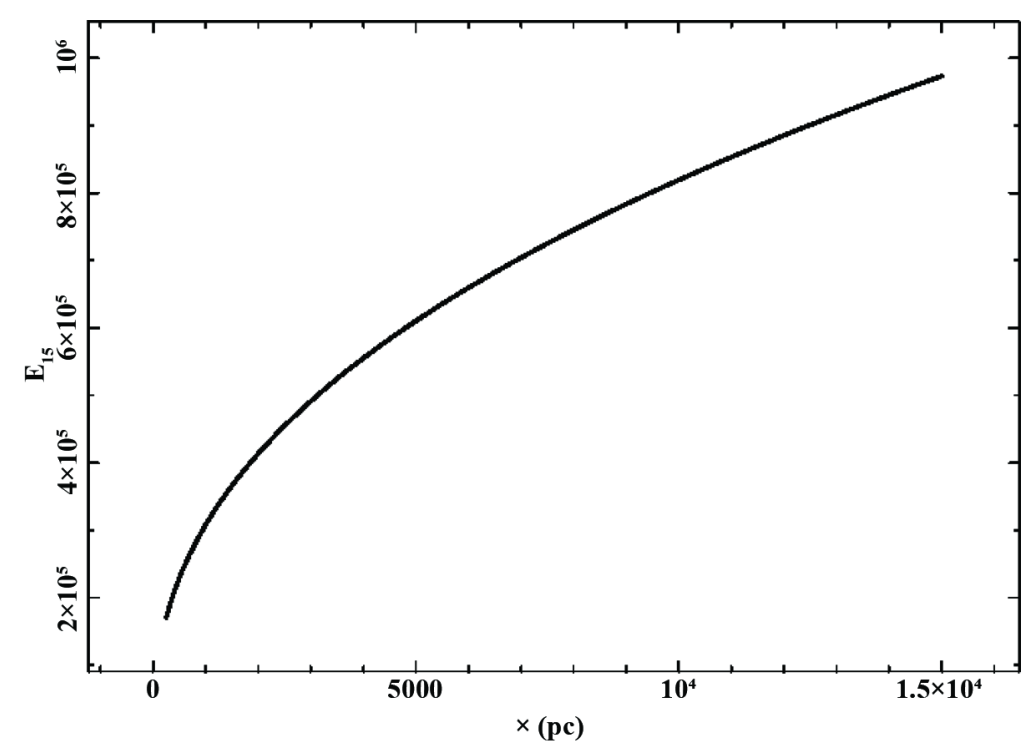

Figure 11. Maximum achievable energy, $E_{15}$, as a function of the distance when $x_{0}=100 \mathrm{pc}, \beta_{0}=0.9, B_{0}=0.025$ gauss, $\alpha=0.1$ and $Z=1$.

two cases. In the first case we have a surrounding medium with constant density and the analytical result is limited to a series expansion for the solution, see Equation (29). In the second case the surrounding density decreases with a power law behaviour and the analytical result is limited to the velocity-distance relation, see Equation (40) and to a series expansion for the solution, see Equation (42).

The losses: The choice of the flux of energy as a quantity to be conserved allows a parametrization of the losses. In the first model we considered the decrease of the available classical and relativistic flux of energy through a recursive relation, see Equation (46) and Equation (49). Figure 6 and Figure 7 show the velocity as a function of the regulating parameter $\varepsilon$. Values of $\varepsilon<0.001$ do not affect the jet's trajectory at the astrophysical distance of $15 \mathrm{kpc}$. In the second model, we fixed a law for the decrease of the available flux of relativistic energy as a function of the distance, see Equation (50) and we derived a law for the decrease of the velocity as a function of the regulating parameter $\eta$, see Figure 8 .

Astrophysical applications: We modeled the behaviour of the magnetic field assuming the conservation of the magnetic flux of energy in the case of constant density, see Equation (51). The availability of an analytical expression for the magnetic field, see the theoretical Equation (53), allows finding a law for the behaviour of the intensity of the synchrotron emission, see Equation (57). The application to the measured intensity of 3C31 yields an efficiency over all the jet's length of $87.56 \%$, see Figure 9. A test on the magnetic field of 3C273 in the pc region can be seen in Figure 10. The presence of a law for the magnetic field allows fixing the Hillas plot for the maximum energy which can reached during the process of acceleration of the cosmic rays, which in the case of $3 \mathrm{C} 31$ is $\approx 10^{21} \mathrm{eV}$, see the caption of Figure 11 .

\section{References}

[1] Reynolds, O. (1883) An Experimental Investigation of the Circumstances Which Determine Whether the Motion of Water Shall Be Direct or Sinuous, and of the Law of Resistance in Parallel Channels. Proceedings of the Royal Society of London, 174, 935-982.

[2] Reynolds, O. (1894) On the Dynamical Theory of Incompressible Viscous Fluids and the Determination of the Criterion. Proceedings of the Royal Society of London, 56, 40-45. http://dx.doi.org/10.1098/rspl.1894.0075

[3] van Dyke, M. (1982) An Album of Fluid Motion. NASA STI/Recon Technical Report A, 82, 36549.

[4] Goldstein, S. (1965) Modern Developments in Fluid Dynamics. Dover, New York.

[5] Landau, L. (1987) Fluid Mechanics. 2nd Edition, Pergamon Press, New York.

[6] Pope, S.B. (2000) Turbulent Flows. Cambridge University Press, Cambridge, UK. http://dx.doi.org/10.1017/CBO9780511840531 
[7] Bird, R., Stewart, W. and Lightfoot, E. (2002) Transport Phenomena. 2nd Edition, John Wiley and Sons, New York.

[8] Lebedev, S.V., Suzuki-Vidal, F., Ciardi, A., Bocchi, M., Bland, S.N., Burdiak, G., Chittenden, J.P., de Grouchy, P., Hall, G.N., Harvey-Thompson, A., Marocchino, A., Swalding, G., Frank, A., Blackman, E.G. and Camenzind, M. (2011) Laboratory Simulations of Astrophysical Jets. In: Bonanno, A., de Gouveia Dal Pino, E. and Kosovichev, A.G., Eds., IAU Symposium vol. 274 of IAU Symposium, 26-35.

[9] Suzuki-Vidal, F., Lebedev, S.V., Krishnan, M., Bocchi, M., Skidmore, J., Swadling, G., Harvey-Thompson, A.J., Burdiak, G., de Grouchy, P., Pickworth, L., Suttle, L., Bland, S.N., Chittenden, J.P., Hall, G.N., Khoory, E., Wilson-Elliot, K., Madden, R.E., Ciardi, A. and Frank, A. (2012) Laboratory Astrophysics Experiments Studying Hydrodynamic and Magnetically-Driven Plasma Jets. Journal of Physics Conference Series, 370, 012002. http://dx.doi.org/10.1088/1742-6596/370/1/012002

[10] De Young, D.S. (2002) The Physics of Extragalactic Radio Sources. University of Chicago Press, Chicago.

[11] Mistry, D. and Dawson, J.R. (2014) Experimental Investigation of Entrainment Processes of a Turbulent Jet. 19th Australasian Fluid Mechanics Conference, Melbourne, 8-11 December 2014, 4 p.

[12] Laing, R.A. and Bridle, A.H. (2002) Relativistic Models and the Jet Velocity Field in the Radio Galaxy 3C 31. MNRAS, 336, 328.

[13] Palacios, A.F. (2015) The Mass-Energy Equivalence Principle in Fluid Dynamics. Journal of High Energy Physics, Gravitation and Cosmology, 1, 48-54. http://dx.doi.org/10.4236/jhepgc.2015.11005

[14] Tenenbaum, M. and Pollard, H. (1963) Ordinary Differential Equations: An Elementary Textbook for Students of Mathematics, Engineering, and the Sciences. Dover Publications, New York.

[15] Ince, E.L. (2012) Ordinary Differential Equations. Courier Dover Publications, New York.

[16] Abramowitz, M. and Stegun, I.A. (1965) Handbook of Mathematical Functions with Formulas, Graphs, and Mathematical Tables. Dover, New York.

[17] Lang, K.R. (1980) Astrophysical Formulae. 2nd Edition, Springer, New York. http://dx.doi.org/10.1007/978-3-662-21642-2

[18] Savolainen, T., Wiik, K., Valtaoja, E. and Tornikoski, M. (2008) Magnetic Field Structure in the Parsec Scale Jet of 3C 273 from Multifrequency VLBA Observations. In: Rector, T.A. and De Young, D.S., Eds., Extragalactic Jets: Theory and Observation from Radio to Gamma Ray Vol. 386 of Astronomical Society of the Pacific Conference Series, 451. (Preprint)

[19] Hillas, A.M. (1984) The Origin of Ultra-High-Energy Cosmic Rays. Annual Review of Astronomy and Astrophysics, 22, 425-444. 